\title{
Associations between peripheral blood lymphocyte subsets and clinical outcomes in patients with lung cancer treated with immune checkpoint inhibitor
}

\author{
Peng $\mathrm{Li}^{1}$, Peng Qin ${ }^{2}$, Xiaomin Fu ${ }^{2}$, Guowei Zhang ${ }^{1}$, Xiangtao Yan ${ }^{1}$, Mina Zhang ${ }^{1}$, Xiaojuan Zhang ${ }^{1}$, \\ Jinpo Yang ${ }^{1}$, Huijuan Wang ${ }^{1}$, Zhiyong $\mathrm{Ma}^{1}$ \\ ${ }^{1}$ Department of Medical Oncology, The Affiliated Cancer Hospital of Zhengzhou University, Henan Cancer Hospital, Zhengzhou, China; \\ ${ }^{2}$ Department of Immunotherapy, The Affiliated Cancer Hospital of Zhengzhou University, Henan Cancer Hospital, Zhengzhou, China \\ Contributions: (I) Conception and design: Z Ma, H Wang, P Li; (II) Administrative support: Z Ma; (III) Provision of study materials or patients: \\ P Qin, X Fu, P Li, Z Ma, H Wang; (IV) Collection and assembly of data: G Zhang, X Yan, M Zhang, X Zhang, J Yang; (V) Data analysis and \\ interpretation: P Li, P Qin, X Fu; (VI) Manuscript writing: All authors; (VII) Final approval of manuscript: All authors. \\ Correspondence to: Zhiyong Ma. Department of Medical Oncology, The Affiliated Cancer Hospital of Zhengzhou University, Henan Cancer Hospital, \\ Dongming Road No.127, Zhengzhou 450008, China. Email: mazhiyong9152@126.com.
}

\begin{abstract}
Background This study aimed to estimate peripheral blood lymphocyte subsets and programmed death receptor-1 positive (PD-1+) proportions of T cells, and their impact on progression free survival (PFS) and radiological response in lung cancer.

Methods: From May 2018 to April 2020, 34patients of the Henan Tumor Hospital who were diagnosed with advanced lung cancer were recruited to this study. Peripheral blood lymphocyte subsets and PD-1+ proportions of $\mathrm{T}$ cells were assessed by flow cytometry before and after treatment with immune checkpoint inhibitors (ICIs). The associations among these parameters, and PFS and clinical response were estimated by survival analysis and Fishers' exact test, respectively.
\end{abstract}

Results: Several lymphocyte variables and biomarkers were found to be correlated with PFS and tumor response, as assessed using the Response Evaluation Criteria in Solid Tumors (RECIST). In all 34 lung cancer participants and a subgroup of 28 participants with non-small cell lung cancer (NSCLC), higher levels of natural killer (NK) cells and higher CD4+/CD8+ cell ratios before the ICIs treatment were associated with longer PFS. Moreover, CD4+ T cells were significantly correlated with radiological response in all 34 lung cancer participants. Of the 28 NSCLC participants, those with higher levels of CD4+ T cells, CD4+/CD8+ cell ratios, absolute numbers of NK cells, and lower levels of regulatory $\mathrm{T}$ cells (Tregs)before treatment had better tumor response. After 2 cycles of combined ICIs treatment, both the absolute numbers of CD4+ $\mathrm{T}$ cells and CD45+ lymphocytes were statistically associated with PFS after being adjusted for gender and neutrophil-lymphocyte ratio (NLR) [hazard ratio $(\mathrm{HR})=0.23, \mathrm{P}=0.015 ; \mathrm{HR}=0.30, \mathrm{P}=0.032$, respectively]. The absolute numbers of CD45+, CD3+, and CD4+ $\mathrm{T}$ lymphocytes were associated with radiological response treated by ICIs $(\mathrm{P}=0.038)$.

Conclusions: Our results suggested that the absolute number of NK cells and CD4+/CD8+ cells ratio before treatment could predict longer PFS and better radiological response in lung cancer patients treated with ICIs combination therapy. In addition, Tregs, as well as the other parameters in lymphocyte subsets, may also predict response.

Keywords: Immune checkpoint inhibitor (ICIs); programmed death receptor-1 (PD-1); lung cancer; peripheral blood lymphocyte subsets

Submitted Dec 10, 2020. Accepted for publication Mar 23, 2021.

doi: $10.21037 /$ apm-21-163

View this article at: http://dx.doi.org/10.21037/apm-21-163 


\section{Introduction}

Over the past few years, immune checkpoint inhibitors (ICIs) have been considered the most significant improvement in tumor treatment. Antibodies against programmed death receptor-1 (PD-1)/PD-1 ligand 1 (PD-L1) showed promising efficiency in certain kinds of solid tumor, including nonsmall cell lung cancer (NSCLC) (1) and small cell lung cancer (SCLC) $(2,3)$. It has been recommended to assess the status of PD-L1 in tumor tissue when considering treatment options for NSCLC (4). Other biomarkers such as tumor mutation burden (TMB) in tumor tissue and in circulating peripheral blood have returned conflicting results on predicting lung cancer treated by ICIs (5). There is a lack of dynamic and longitudinal methods to monitor the efficiency of ICIs, and developing such methods may become the main focus of study for ICIs.

Liquid biopsy was considered a suitable non-invasive and dynamic method to predict and evaluate the clinical outcomes of ICIs. The strategy for identifying tumorreactive $\mathrm{T}$ cells in peripheral blood is to focus on the cytotoxic CD8+ T cells that express PD-1, a well-known marker for previously activated or exhausted $\mathrm{T}$ cells. Tumor neoantigen-specific $\mathrm{T}$ cell subpopulations could be isolated from the peripheral blood of tumor patients (6-8). Besides, a recent study indicated that the distribution of peripheral blood lymphocyte subsets and expression of PD-1 on T cells before treatment might predict the outcome of Nivolumab in NSCLC patients. Assessing the pre-treatment levels of exhausted $\mathrm{T}$ cells as well as their decrease upon treatment may also predict response and clinical outcome (9). Chemotherapy might also regulate the proportions of immune cells in peripheral blood (10). Some preclinical studies have suggested that oral anti-angiogenic agents could optimize the efficiency of ICIs $(11,12)$. The combination of ICIs with chemotherapy or oral antiangiogenic agents has promising potential for the clinical treatment of tumors in the clinical practice $(1,13-15)$. The potential prediction roles of peripheral blood lymphocyte subsets and expression of PD-1 on T cells are still unknown and need to be clarified for lung cancer patients treated by ICIs in combination therapies.

To estimate the potential prediction of peripheral blood lymphocyte subsets on lung cancer patients using ICIs combinations in the real world, flow cytometer was used to detect 12 parameters before and after the ICIs treatment. We hypothesized that the proportions of peripheral blood lymphocyte subsets and PD-1 expression on T cells during treatment may help to predict the clinical outcomes in lung cancer patients. In the present study, we performed flow cytometry analysis to investigate the different lymphocyte subsets before and after ICIs treatment, including ICIs combined with anti-angiogenic agents, ICIs combined with chemotherapy, and so on. We present the following article in accordance with the MDAR checklist (available at http:// dx.doi.org/10.21037/apm-21-163).

\section{Methods}

\section{Study population and assessments of clinical outcomes}

This retrospective study included patients with advanced NSCLC who were receiving PD-1 inhibitors in the Medical Oncology and Immunotherapy Departments of the Henan Tumor Hospital from May 2018 to April 2020. The main eligibility criteria for treatment with PD-1 inhibitors (including pembrolizumab, nivolumab, sintilimab, camrelizumab, tislelizumab, and toripalimab) included histological diagnosis of advanced lung cancer (stage IV NSCLC and extensive stage SCLC), Eastern Cooperative Oncology Group Performance Status (ECOG-PS) $\leq 2$. The major exclusion criteria were the need for systemic corticosteroid treatment at a dose $>10 \mathrm{mg}$ /day of prednisone (or equivalent), and the uncontrolled brain metastases. Eligible patients could have received PD-1 inhibitor alone, chemotherapy combined with PD-1 inhibitor, or oral antiangiogenesis drugs (including anlotinib and apatinib). The neutrophil-lymphocyte ratio (NLR) was defined as the neutrophil count divided by the lymphocyte count, and was included on account of previous support for its prognostic effect (16). After exclusion of patients with incomplete follow-up information, a total of 34 participants remained for the PFS analysis. This retrospective study was approved by the institutional review board of Henan Tumor Hospital and performed in compliance with Helsinki Declaration (as revised in 2013). Before the initiation of any study-related procedures, written informed consent was given by each participant.

\section{Response assessment}

The participants underwent a therapeutic effect assessment every 2 cycles of PD-1 inhibitor. Radiological response assessment was performed using the Response Evaluation Criteria in Solid Tumors (RECIST) v. 1.1; moreover, because RECIST may underestimate the evaluation of therapeutic effect of ICIs, an extra response assessment was performed using the immune-related response criteria 
(irRC). The best overall response (BOR) during the whole treatment with ICIs was recorded for both RECIST and irRC according to the following groups: complete response (CR), partial response (PR), stable disease (SD), and progressive disease (PD). For purposes of our analyses, participants were then allocated to: (I) responder group, which included all patients with CR or PR or (II) no responder group, which included all patients with $\mathrm{SD}$ or PD. The PFS was calculated from the date that treatment began to the first instance of tumor progression according to RECIST (RECIST-PFS) or irRC (irRC-PFS), date at last clinical examination, date of death of any cause before tumor progression, or the last contact date.

\section{Blood collection and flow cytometry}

Peripheral blood cells $(2 \mathrm{~mL})$ were collected from participants who had provided written informed consent before their first treatment with a PD-1 inhibitor. A total of 3 panels were designed for flow cytometry. The CD3-48 panel was used to detect the ratio of $\mathrm{CD} 3, \mathrm{CD} 4$, and $\mathrm{CD} 8$ in $\mathrm{CD} 45+$ lymphocytes and the expression of PD-1 on these cells. In the $\mathrm{NK}+\mathrm{B}+$ Trucount panel, BD Trucount tubes (Becton, Dickinson and Co., San Jose, CA, USA) were used to detect the proportion and absolute counts of NK and $\mathrm{B}$ cells in peripheral blood lymphocytes; meanwhile, the absolute counts of CD45+ lymphocytes and CD3+ T cells were determined in this panel. Finally, regulatory $\mathrm{T}$ cells (Tregs) in CD4+ $\mathrm{T}$ cells was detected in the Tregs panel with the phenotype of CD25+ and CD127. According to the absolute count of $\mathrm{CD} 3+\mathrm{T}$ cells in the $\mathrm{NK}+\mathrm{B}+$ Trucount panel and the proportion of CD4+and CD8+T cells in $\mathrm{CD} 3+\mathrm{T}$ cells in the CD3-4-8 panel, we calculated the absolute number of CD4+ and CD8+ T cells in peripheral blood. The representative gating strategy and the expression of PD-1 on CD45+ T cells, CD3+ T cells, CD8+ T cells, $\mathrm{CD} 4+\mathrm{T}$ cells from one representative patient were shown in Figure 1. The detailed operational procedures and related materials are provided in the Supplementary documents.

\section{Statistical analysis}

Descriptive statistics were used to summarize the investigatory findings of the 12 variables tested from peripheral blood lymphocytes and participant characteristics. The median values were set as the cutoff values for the higher and lower groups. The median survival time was calculated using the Kaplan-Meier method, and the survival curves were compared using log-rank test. Multivariate Cox proportional hazards regression models were conducted to test the effect of each variable [in terms of hazards ratios (HRs) and their corresponding 95\% confidence intervals (CIs)] on PFS with or without adjustment for selected factors by using the univariate Cox proportional hazards regression models (for PFS, 2 variables were adjusted: gender and NLR). All tests were 2 -sided, and a $\mathrm{P}$ value $<0.05$ was considered statistically significant. All data were analyzed with $\mathrm{R}$ version 4.0.3 software (https://www.r-project.org/), if not mentioned elsewhere. The survival curve and box plot were performed by Prism GraphPad Software 8.0(San Diego, CA, USA).

\section{Results}

\section{Participant characteristics and clinical outcomes}

A total of 34 patients were enrolled in this study. The baseline clinical and histology characteristics are listed in Table 1. Epidermal growth factor receptor EGFR gene mutations were detected by next-generation sequencing (NGS) in 11 participants and only 2 participants were EGFR gene sensitive mutation positive. Both EGFR gene mutation participants received ICIs after the EGFRtyrosine kinase inhibitor (TKI) treatment and did not have T790M mutation. The protein expressions of PD-L1 were tested by immunohistochemistry in 14 participants, of which 9 were positive. The tumor response rate was $33.3 \%$ (3 PR), the median PFS was 17.8 months in the 9 positive patients; and 20\% (1 PR) and 12.3 months in the 5 negative patients. These results were not significant statistically for the small sample. In this study, 10 patients had immune related adverse events, including immune related hypothyroidism $(\mathrm{n}=6)$, immune related livery injury $(\mathrm{n}=1)$, immune related thrombocytopenia $(n=1)$, immune related hyperthyroidism $(\mathrm{n}=1)$, immune related skin rash $(\mathrm{n}=1)$.

The median number of ICI administrations was 6 cycles, with a range of 2-17 cycles. Overall, 6 participants had died at the last contact. The median PFS and overall survival (OS) were 9.95 and 24.07 months, respectively. The best of response in RECIST was reported as follows: $P R=19$, $\mathrm{SD}=10, \mathrm{PD}=5$; no complete responses were observed. The associations between the 34 participant characteristics and PFS were tested by univariate and multivariate analysis. However, we did not find statistical significance in the univariate analysis (data not shown). In the multivariate analysis, gender and NLR were significantly associated with 


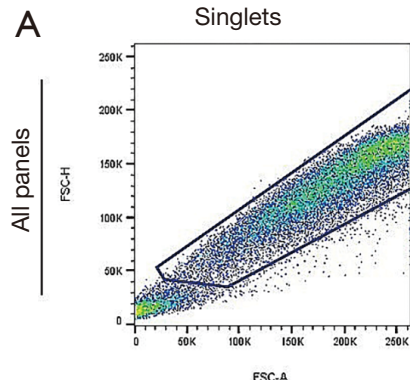

FSC-A

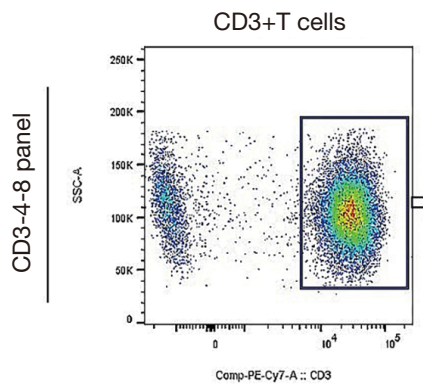

COMP-PE-CY7-A:: CD3

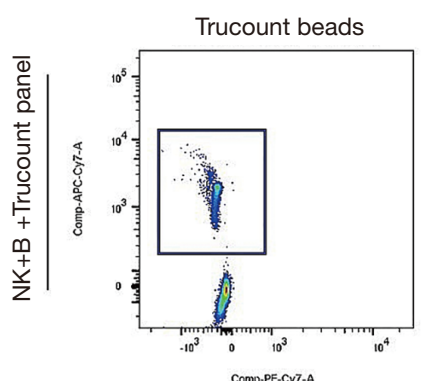

Comp-PE-Cy7-A

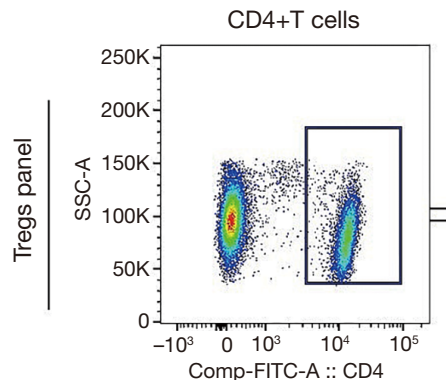

CD45+ lymphocytes
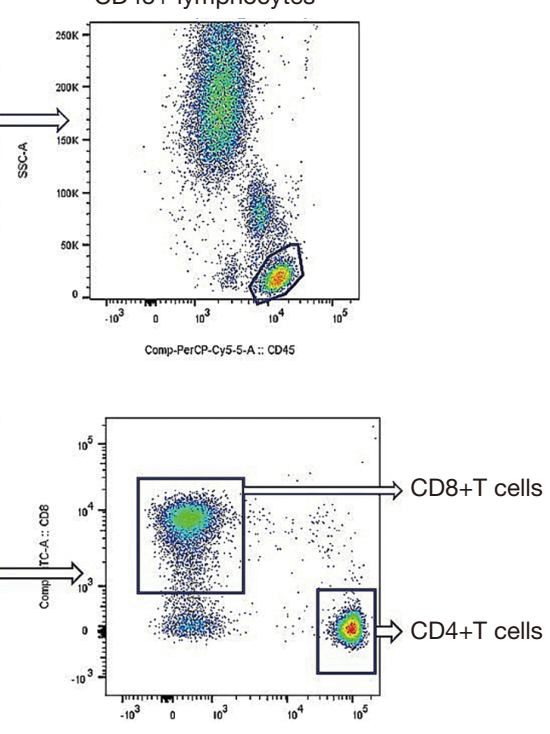

COMPP.PE-A: CD4
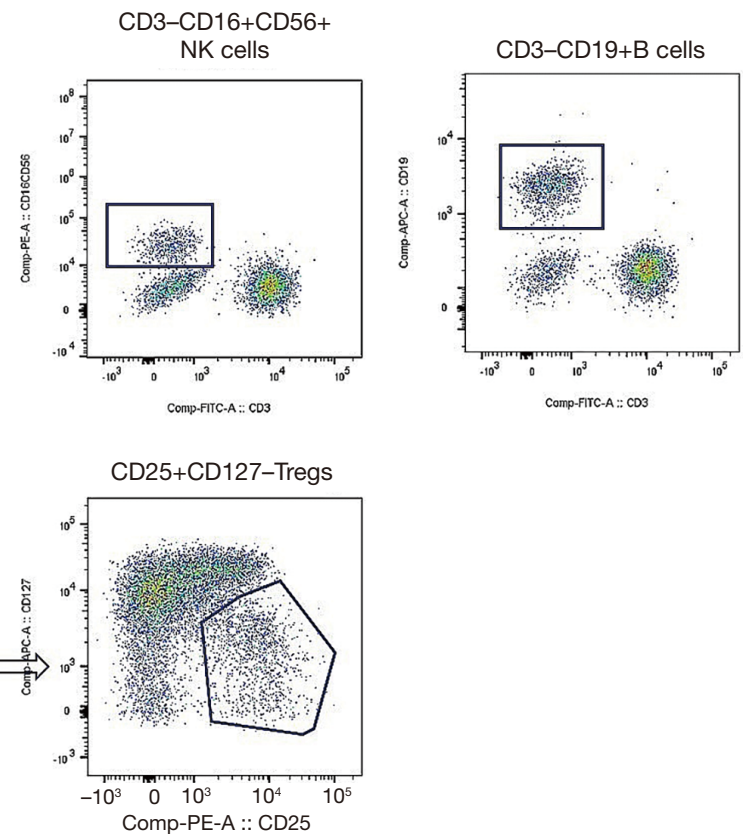

B
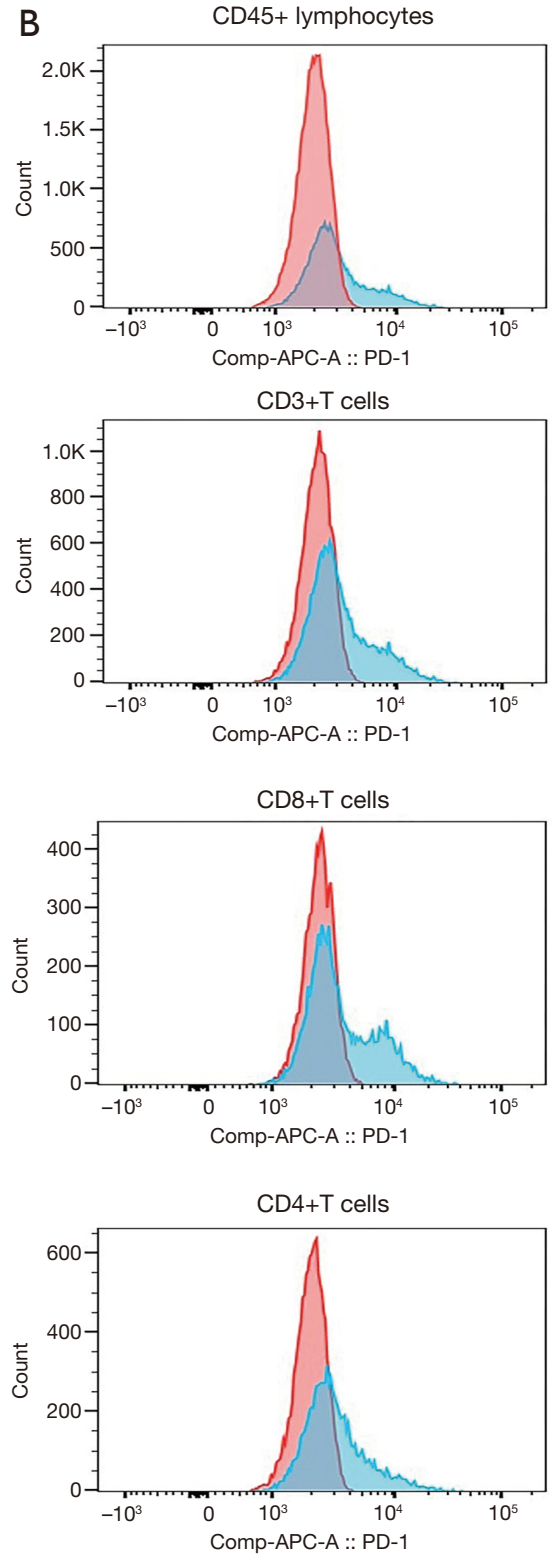

PD-1-ISO

PD-1

Figure 1 Identification the subgroups and PD-1 expression of lymphocytes in blood of lung cancer participants by flow cytometry. Flow cytometry of lymphocytes in blood of lung cancer participants (A) Representative gating strategy: As shown in the top row, all data files except the bead events gated for absolute counts of lymphocytes were first preprocessed to remove adhesion cells by FSC-A and FSC-H and then circle the CD45+ lymphocytes. CD3-4-8 panel was used to identify CD3+, CD4+ and CD8+ T cells. NK+B+ Trucount panel was used for identifying NK and B cells and determining absolute counts of lymphocytes in bloods. Tregs panel was used to characterize Tregs in CD4+T cells (CD4+CD25+CD127-). (B) The expression of PD-1 on CD45+ T cells, CD3+ T cells, CD8+ T cells, CD4+ T cells from one representative patient. 
Table 1 Summary of clinical and pathological participant characteristics

\begin{tabular}{lcc}
\hline Participant characteristic & $\mathrm{N}$ & $\%$ \\
\hline Gender & 28 & 82.4 \\
Male & 6 & 17.6 \\
Female & & \\
Age & 18 & 52.9 \\
$<65$ & 16 & 47.1 \\
$\geq 65$ & &
\end{tabular}

Smoker

Yes

No

Histology

Adenocarcinoma
Squamous cell lung cancer
Small cell lung cancer

Treatment line

\section{First and second line}

Third line and beyond

Brain metastasis

$\begin{array}{ll}\text { No } & 24 \\ \text { Yes } & 10\end{array}$

Live metastasis

No

Yes

Bone metastasis

No

Yes

Treatment model

$\mathrm{ICls}$ alone

ICls plus

Best treatment response

\section{Response}

No response

NLR

$\begin{array}{ll}\text { Low } & 17 \\ \text { High } & 17\end{array}$

ICls, immune checkpoint inhibitors; NLR, neutrophil-lymphocyte ratio.
PFS (Figure S1). Hence, in the subsequent analysis, gender and NLR were selected as adjusted factors in multivariable analysis.

\section{Peripheral blood lymphocytes subsets and PFS in 34 lung cancer patients and 28 NSCLC patients}

We examined 12 variables in the peripheral blood lymphocytes tests of 34 lung cancer participants, including Tregs, B cells, NK cells, CD45+ T cells, CD8+ T cells, CD4+ T cells, CD3+ T cells, CD4+/CD8+ ratio, PD1+/CD45+ T cells, PD-1+/CD8+ T cells, PD-1+/CD8+ $\mathrm{T}$ cells, $\mathrm{PD}-1+/ \mathrm{CD} 4+\mathrm{T}$ cells, and $\mathrm{PD}-1+/ \mathrm{CD} 3+\mathrm{T}$ cells. In the survival analysis, when $\mathrm{HR}<1$, the variable was a protective factor, which meant that groups with higher numbers had longer survival outcomes. The associations between the 12 variables in peripheral blood lymphocyte tests and PFS were not significant in the univariable analysis (data no shown). However, the absolute count of NK cells and CD4+/CD8+ ratio were significantly associated with PFS by multivariable analysis (forest plot shown in Figure $2 A$ ). The analysis of association between NK cell absolute counts and PFS by Kaplan-Meier survival curve is shown in Figure $2 B$. In the log-rank test, the median PFS in groups with lower NK cells was 8.63 months, and the higher group was 17.83 months $(\mathrm{P}=0.008)$. The KaplanMeier survival curve analysis of the association between CD4+/CD8+ cells ratio and PFS is shown in Figure 2C. In the log-rank test, the median PFS in the lower grouping of CD4+/CD8+ T cells ratio was 8.73 months, and the higher grouping was 11.53 months $(\mathrm{P}=0.017)$.

Among the 28 NSCLC participants, the association between the 12 variables in the peripheral blood lymphocytes test and PFS were not significant in the univariable analysis (data not shown). However, the absolute counts of $\mathrm{NK}$ cells and $\mathrm{CD} 4+/ \mathrm{CD} 8+$ ratio were significantly associated with PFS by multivariable analysis (forest plot shown in Figure $3 A$ ). The KaplanMeier survival curve analysis of the association between the absolute count of NK cells and PFS is shown in Figure $3 B$. In the log-rank test, the median PFS in the contingent with lower counts of NK cells was 8.68 months, and the higher group was 18.37 months $(\mathrm{P}=0.013)$. KaplanMeier survival curve analysis of the association between CD4+/CD8+ cells ratio and PFS is shown in Figure 3C. In the log-rank test, the median PFS in the lower group of $\mathrm{CD} 4+/ \mathrm{CD} 8+\mathrm{T}$ cells ratio was 8.73 months, and the higher group was 18.37 months $(\mathrm{P}=0.016)$. 
A

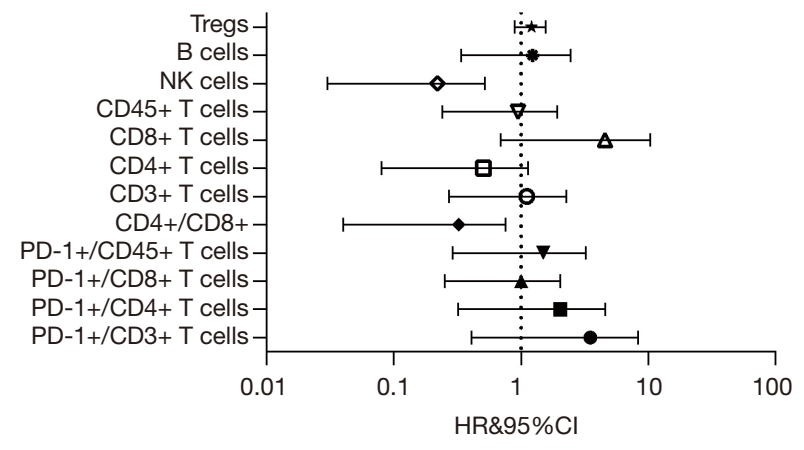

- PD-1+/CD3+ T cells

- PD-1+/CD4+ T cells

^ PD-1+/CD8+ T cells

$\checkmark \mathrm{PD}-1+/ \mathrm{CD} 45+\mathrm{T}$ cells

- CD4+/CD8+

- CD3+ T cells

口 CD4+ T cells

$\Delta$ CD8+ T cells

$\boldsymbol{\nabla}$ CD45+ T cells

$\diamond$ NK cells

- B cells

$\star$ Tregs

B

PFS survival curve by NK cells $(\mathrm{N}=34)$

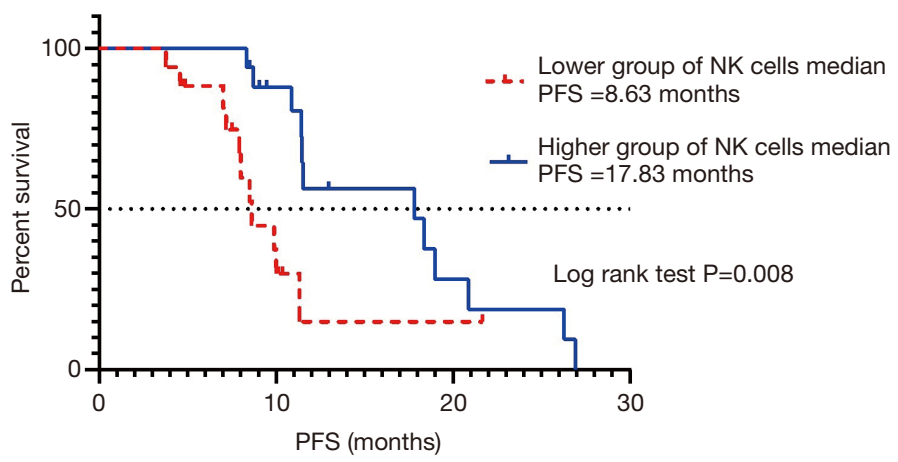

C

PFS survival curve by CD4+/CD8+ T cells ratio $(\mathrm{N}=34)$

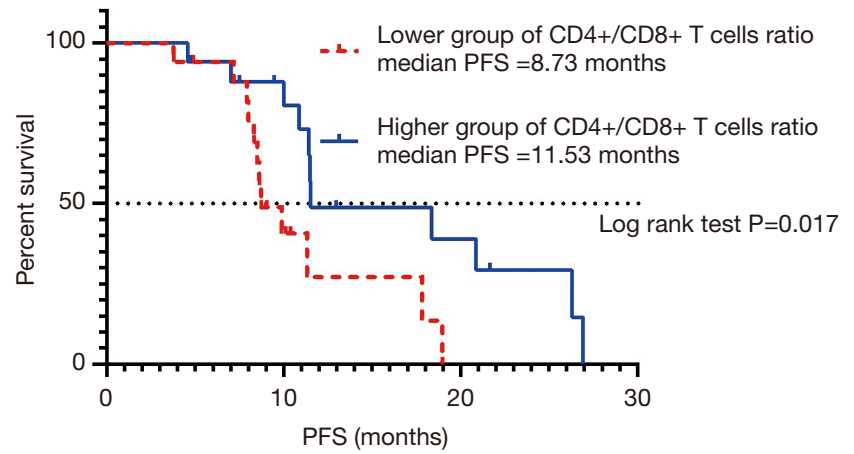

Figure 2 The associations between peripheral blood lymphocyte subsets and PFS in 34 lung cancer participants. PFS, progression free survival; NK, natural killer.

\section{Peripheral blood lymphocyte subsets and radiological response in all lung cancer participants and NSCLC participants}

Next, we investigated the correlation between baseline peripheral blood lymphocyte subsets and radiological response. Notably, analyses using the irRC criteria did not show any differences from those using RECIST 1.1. Among the 34 lung cancer participants, only the absolute value of CD4+ $T$ cells at baseline was profoundly associated with radiological response by unpaired t-test $(\mathrm{P}=0.018)$, and is shown in Figure 4.

Among the 28 NSCLC participants, in agreement with 


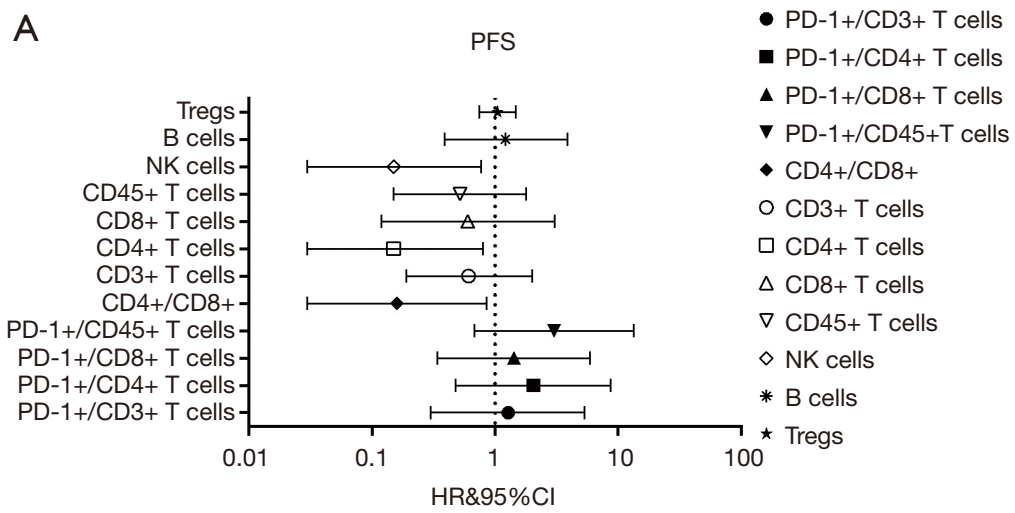

B

PFS survival curve by NK cells $(\mathrm{N}=28)$

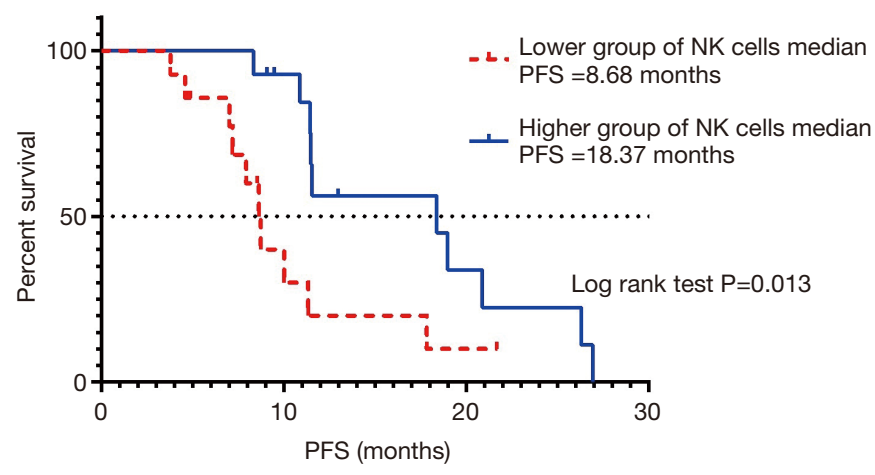

C

PFS survival curve by $\mathrm{CD} 4+/ \mathrm{CD} 8+\mathrm{T}$ cells ratio $(\mathrm{N}=28)$

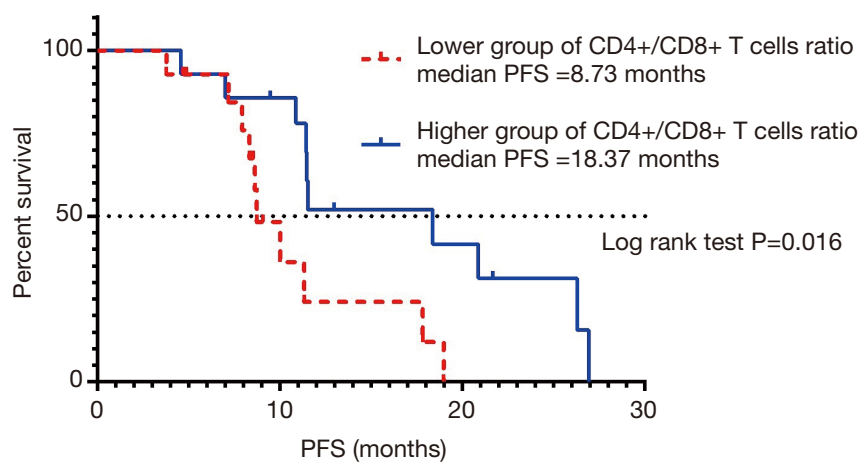

Figure 3 The associations between peripheral blood lymphocytes subsets and PFS in 28 NSCLC patients. PFS, progression free survival; NSCLC, non-small cell lung cancer.

our data correlating peripheral blood lymphocyte subsets and PFS, higher numbers of NK cells and CD4+/CD8+ ratios were statistically associated with radiological response by unpaired t-test $(\mathrm{P}=0.006$ and $\mathrm{P}=0.030$, respectively).
In addition, higher grouping of CD4+ $\mathrm{T}$ cells and lower grouping of Tregs were significantly associated with radiological response in the unpaired t-test $(\mathrm{P}=0.008$ and $\mathrm{P}=0.019$ ), respectively (Figure 5). 


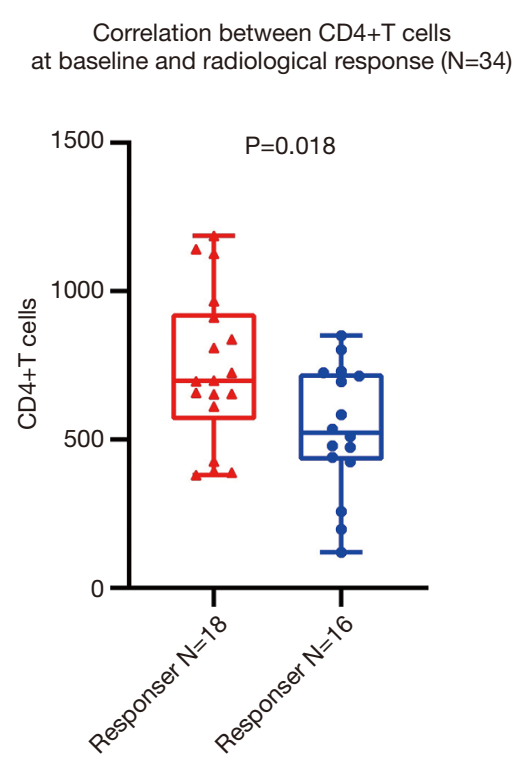

Figure 4 Correlation between CD4+ $\mathrm{T}$ cells at baseline and radiological response in 34 lung cancer participants.

\section{Peripheral blood lymphocyte subsets and clinical outcomes (PFS and radiological response) in all lung cancer and NSCLC participants after 2 cycles of ICI treatments}

The peripheral blood lymphocyte subsets of 33 participants were assessed after 2 cycles of ICIs treatment. In the univariate analysis, no variables were associated with PFS. In the multivariate analysis, the absolute number of $\mathrm{CD} 4+$ $\mathrm{T}$ cells and CD45+ lymphocytes were associated with PFS statistically adjusted by gender and NLR ( $H R=0.23$, $\mathrm{P}=0.015 ; \mathrm{HR}=0.30, \mathrm{P}=0.032$, respectively).

Due to the small sample size, Fisher's exact test was used to analyze the associations between radiological response and lymphocyte subsets after treatment. The absolute numbers of $\mathrm{CD} 45+, \mathrm{CD} 3+$, and $\mathrm{CD} 4+\mathrm{T}$ lymphocytes were associated with response to treatment with ICIs $(\mathrm{P}=0.038)$.

\section{Discussion}

In this real-world study, we found that several variables in peripheral blood lymphocyte subsets tested prior to treatment were associated with clinical outcomes in lung cancer participants treated with ICIs. A total of 34 lung cancer patients were enrolled including 28 NSCLC patients, and they were treated with ICIs alone or ICIs combined therapy. Among the entire participant cohort, compared to those with lower levels of NK cells, participants with higher levels of NK cells had longer PFS. We also found that the CD4+/CD8+ cells ratio was associated with PFS in the entire participant group. Participants with higher CD4+/CD8+ cell ratios were found to have longer PFS. Furthermore, CD4+ T cells at baseline were significantly correlated with the radiological response in the 34 lung cancer participants. The participants with higher levels of CD4+ T cells, CD4+/CD8+ ratio, absolute numbers of NK cells, and lower levels of Tregs had better tumor responses.

The CD4+/CD8+ ratio in peripheral blood lymphocytes is an old marker in many diseases (17), and is also associated with lung cancer risk (18). Low CD4+/CD8+ ratio has been correlated with high-risk types of lung cancer (19). In a small retrospective study (20), an increasing lymphocyte ratio was associated with disease control in NSCLC patients treated with an ICIs alone. In present study, the median CD4+/ CD8+ ratio of the 34 lung cancer participants was 1.44 (0.3-3.6). A higher CD4+/CD8+ ratio was associated with better PFS. In the 28 NSCLC participants, tumor response was also correlated with a higher $\mathrm{CD} 4+/ \mathrm{CD} 8+$ ratio.

A similar study reported on 74 participants in an advanced NSCLC cohort who were treated with nivolumab and had their immune parameters assessed from peripheral blood lymphocyte subsets during treatment. They found that higher levels of CD3+, CD4+, and CD8+ T cells but lower levels of NK cells at baseline was associated with longer OS (9). In the present study, participants with higher levels of CD4+ had a trend of longer PFS but this trend was not statistically significant. Conversely, the baseline absolute numbers of NK in peripheral blood were suggested to be positively associated with longer PFS in our investigation. This data is similar to a previous report by Mazzaschi et al. (21). The NK cells are innate cytotoxic lymphocytes that actively prevent tumor development, growth, and metastatic dissemination in a process called cancer immunosurveillance; some chemotherapies have been created to regulate the functions of NK cells for anti-tumor immunity (22). Yang et al. reported that anlotinib (11), an oral multi-targeted anti-angiogenic agent, could reprogram the tumor immune microenvironment in a manner that augments anti-cancer innate immune cells, including NK cells, dendritic cells (DC), and so on. Moreover, this kind of combination might have potential additive or synergistic anti-tumor efficacy.

In the NSCLC subgroup analysis, higher levels of CD4+/CD8+ ratio, CD4+, and NK cells at baseline were associated with higher PFS, and parallel evaluation of the 3 variables was correlated with higher response. However, a 

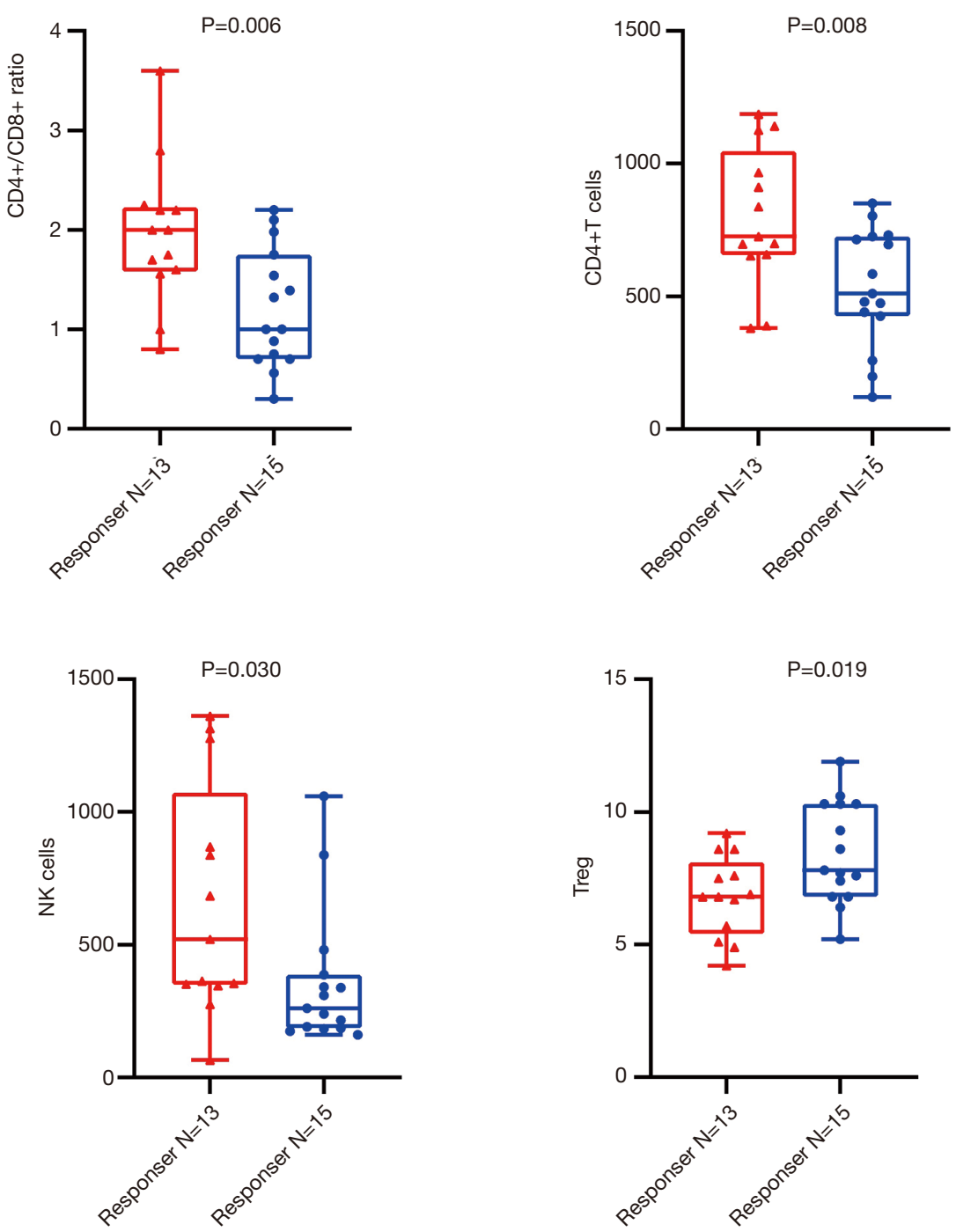

Figure 5 Correlation between CD4+/CD8+ ratio, CD4+ T cells, NK cells and Treg cells at baseline and radiological response in 28 lung cancer participants. NK, natural killer; Treg, T regulatory.

higher level of Treg was correlated with worse radiological response, which was not associated with PFS. These findings could inspire further investigation at the tumor site as opposed to the peripheral blood lymphocyte subsets.

Remarkably, our results were derived from real word clinical practice, the participants were treated with different combinations with ICIs including single ICIs $(\mathrm{n}=7)$, double ICIs $(\mathrm{n}=1)$, chemotherapy plus ICIs $(\mathrm{n}=6)$, and small molecular antiangiogenic drug plus ICIs $(n=20)$. Among the 34 participants, only 14 were evaluated for the expression of PD-L1 protein in tumor tissues, of which 9 were positive and 5 were negative. On account of our small sample size, we did not perform sub-group analyses based on histology and smoking status. We proceeded with multivariable analysis in Cox regression models for PFS. The NLR and gender were the major factors of adjustment. In addition, the PD-1 positive $\mathrm{T}$ cells at baseline were not associated with the PFS and response in 34 lung cancer participants based on peripheral blood lymphocyte subsets analysis, which was contrary to the findings of Ottonello et al. (9). Results from metastatic melanoma samples indicate that a higher proportion of $\mathrm{PD}-1+/ \mathrm{CD} 8+$ among tumor infiltrating lymphocytes (TILs) predicts higher response and longer PFS (23).

Nevertheless, the absolute numbers of circulating NK cells and the CD4+/CD8+ ratio were the main factors 
affecting the PFS in cases of lung cancer receiving different ICIs combinations.

Our study had some limitations. Firstly, the participants had different kinds of treatment combinations and the sample size was small. Studies involving larger sample sizes are required to validate our results. Secondly, our present data were based on the peripheral blood lymphocyte subsets analysis at the baseline, so it might not have reflected the actual situations at the primary tumor site. And lack of further longitudinal peripheral blood samples collecting. Thirdly, the benefit to OS was the primary goal of immunotherapy for the lung cancer, and ongoing subsequent follow-up is required. Fourthly, the ICIs and treatment combinations were from different pharmaceutical corporations may be an important confounder in the analysis.

Currently, the expression levels of PD-L1protein in lung cancer tissue are defined as the main positive biomarker in clinical practice $(24,25)$. Besides, using tumor mutation burden (TMB) in tumor tissue or peripheral blood has yielded mixed results in lung cancer, and still needs further assessment before applying it to clinical practice $(26,27)$. Recently, STK11/LKB1 was considered a negative biomarker in ICIs treatments, regardless of KRAS status $(28,29)$. Further investigations might focus on the tissuebased biomarkers and circulating blood, including the peripheral blood lymphocyte subsets analysis as in our study.

In spite of these limitations, our small sample size lung cancer study, mainly investigating treatment with ICIs plus small molecular anti-angiogenic drugs, provided novel immune parameters to potentially predict clinical outcomes based on the analysis of peripheral blood lymphocyte subsets.

\section{Acknowledgments}

We thank all patients and investigators for their participation in this study.

Funding: 2018 Henan Medical Science and Technology Public Relations Project, No. 2018020507.

\section{Footnote}

Reporting Checklist: The authors have completed the MDAR checklist. Available at http://dx.doi.org/10.21037/apm-21-163

Data Sharing Statement: Available at http://dx.doi. org/10.21037/apm-21-163

Conflicts of Interest: All authors have completed the ICMJE uniform disclosure form (available at http://dx.doi. org/10.21037/apm-21-163). The authors have no conflicts of interest to declare.

Ethical Statement: The authors are accountable for all aspects of the work in ensuring that questions related to the accuracy or integrity of any part of the work are appropriately investigated and resolved. This retrospective study was approved by the institutional review board of Henan Tumor Hospital and performed in compliance with Helsinki Declaration (as revised in 2013). Before the initiation of any study-related procedures, written informed consent was given by each participant.

Open Access Statement: This is an Open Access article distributed in accordance with the Creative Commons Attribution-NonCommercial-NoDerivs 4.0 International License (CC BY-NC-ND 4.0), which permits the noncommercial replication and distribution of the article with the strict proviso that no changes or edits are made and the original work is properly cited (including links to both the formal publication through the relevant DOI and the license). See: https://creativecommons.org/licenses/by-nc-nd/4.0/.

\section{References}

1. Kim R, Keam B, Hahn S, et al. First-line Pembrolizumab Versus Pembrolizumab Plus Chemotherapy Versus Chemotherapy Alone in Non-small-cell Lung Cancer: A Systematic Review and Network Meta-analysis. Clin Lung Cancer 2019;20:331-8.e4.

2. Paz-Ares L, Dvorkin M, Chen $Y$, et al. Durvalumab plus platinum-etoposide versus platinum-etoposide in firstline treatment of extensive-stage small-cell lung cancer (CASPIAN): a randomised, controlled, open-label, phase 3 trial. Lancet 2019;394:1929-39.

3. Nishio M, Sugawara S, Atagi S, et al. Subgroup Analysis of Japanese Patients in a Phase III Study of Atezolizumab in Extensive-stage Small-cell Lung Cancer (IMpower133). Clin Lung Cancer 2019;20:469-76.e1.

4. Melosky B, Chu Q, Juergens RA, et al. Breaking the biomarker code: PD-L1 expression and checkpoint inhibition in advanced NSCLC. Cancer Treat Rev 2018;65:65-77.

5. Proto C, Ferrara R, Signorelli D, et al. Choosing wisely first line immunotherapy in non-small cell lung cancer (NSCLC): what to add and what to leave out. Cancer Treat Rev 2019;75:39-51.

6. Rizvi NA, Hellmann MD, Snyder A, et al. Cancer 
immunology. Mutational landscape determines sensitivity to PD-1 blockade in non-small cell lung cancer. Science 2015;348:124-8.

7. Gros A, Parkhurst MR, Tran E, et al. Prospective identification of neoantigen-specific lymphocytes in the peripheral blood of melanoma patients. Nat Med 2016;22:433-8.

8. Schumacher TN, Scheper W. A liquid biopsy for cancer immunotherapy. Nat Med 2016;22:340-1.

9. Ottonello S, Genova C, Cossu I, et al. Association Between Response to Nivolumab Treatment and Peripheral Blood Lymphocyte Subsets in Patients With Non-small Cell Lung Cancer. Front Immunol 2020;11:125.

10. de Goeje PL, Poncin M, Bezemer K, et al. Induction of Peripheral Effector CD8 T-cell Proliferation by Combination of Paclitaxel, Carboplatin, and Bevacizumab in Non-small Cell Lung Cancer Patients. Clin Cancer Res 2019;25:2219-27.

11. Yang Y, Li L, Jiang Z, et al. Anlotinib optimizes antitumor innate immunity to potentiate the therapeutic effect of PD-1 blockade in lung cancer. Cancer Immunol Immunother 2020;69:2523-32.

12. Cai X, Wei B, Li L, et al. Apatinib enhanced anti-PD-1 therapy for colon cancer in mice via promoting PD-L1 expression. Int Immunopharmacol 2020;88:106858.

13. Zhao S, Ren S, Jiang T, et al. Low-Dose Apatinib Optimizes Tumor Microenvironment and Potentiates Antitumor Effect of PD-1/PD-L1 Blockade in Lung Cancer. Cancer Immunol Res 2019;7:630-43.

14. Sheng X, Yan X, Chi Z, et al. Axitinib in Combination With Toripalimab, a Humanized Immunoglobulin G4 Monoclonal Antibody Against Programmed Cell Death-1, in Patients With Metastatic Mucosal Melanoma: An OpenLabel Phase IB Trial. J Clin Oncol 2019;37:2987-99.

15. Xu J, Zhang Y, Jia R, et al. Anti-PD-1 Antibody SHR-1210 Combined with Apatinib for Advanced Hepatocellular Carcinoma, Gastric, or Esophagogastric Junction Cancer: An Open-label, Dose Escalation and Expansion Study. Clin Cancer Res 2019;25:515-23.

16. Zer A, Sung MR, Walia P, et al. Correlation of Neutrophil to Lymphocyte Ratio and Absolute Neutrophil Count With Outcomes With PD-1 Axis Inhibitors in Patients With Advanced Non-Small-Cell Lung Cancer. Clin Lung Cancer 2018;19:426-34.e1.

17. Amadori A, Zamarchi R, De Silvestro G, et al. Genetic control of the CD4/CD8 T-cell ratio in humans. Nat Med 1995;1:1279-83.

18. Clifford GM, Lise M, Franceschi S, et al. CD4/CD8 ratio and lung cancer risk. Lancet HIV 2017;4:e103.

19. Sigel K, Wisnivesky J, Crothers K, et al. Immunological and infectious risk factors for lung cancer in US veterans with HIV: a longitudinal cohort study. Lancet HIV 2017;4:e67-73.

20. Zhuo M, Chen H, Zhang T, et al. The potential predictive value of circulating immune cell ratio and tumor marker in atezolizumab treated advanced non-small cell lung cancer patients. Cancer Biomark 2018;22:467-76.

21. Mazzaschi G, Facchinetti F, Missale G, et al. The circulating pool of functionally competent $\mathrm{NK}$ and CD8+ cells predicts the outcome of anti-PD1 treatment in advanced NSCLC. Lung Cancer 2019;127:153-63.

22. Zingoni A, Fionda C, Borrelli C, et al. Natural Killer Cell Response to Chemotherapy-Stressed Cancer Cells: Role in Tumor Immunosurveillance. Front Immunol 2017;8:1194.

23. Daud AI, Loo K, Pauli ML, et al. Tumor immune profiling predicts response to anti-PD-1 therapy in human melanoma. J Clin Invest 2016;126:3447-52.

24. Reck M, Rodriguez-Abreu D, Robinson AG, et al. Pembrolizumab versus Chemotherapy for PD-L1Positive Non-Small-Cell Lung Cancer. N Engl J Med 2016;375:1823-33.

25. Sun Y, Zhai C, Chen X, et al. Characterization of PD-L1 protein expression and CD8(+) tumor-infiltrating lymphocyte density, and their associations with clinical outcome in smallcell lung cancer. Transl Lung Cancer Res 2019;8:748-59.

26. Chae YK, Davis AA, Agte S, et al. Clinical Implications of Circulating Tumor DNA Tumor Mutational Burden (ctDNA TMB) in Non-Small Cell Lung Cancer. Oncologist 2019;24:820-8.

27. Boumber Y. Tumor mutational burden (TMB) as a biomarker of response to immunotherapy in small cell lung cancer. J Thorac Dis 2018;10:4689-93.

28. Laderian B, Mundi P, Fojo T, et al. Emerging Therapeutic Implications of STK11 Mutation: Case Series. Oncologist 2020;25:733-7.

29. Arora S, Velichinskii R, Lesh RW, et al. Existing and Emerging Biomarkers for Immune Checkpoint Immunotherapy in Solid Tumors. Adv Ther 2019;36:2638-78.

Cite this article as: Li P, Qin P, Fu X, Zhang G, Yan X, Zhang M, Zhang X, Yang J, Wang H, Ma Z. Associations between peripheral blood lymphocyte subsets and clinical outcomes in patients with lung cancer treated with immune checkpoint inhibitor. Ann Palliat Med 2021;10(3):3039-3049. doi: 10.21037/apm-21-163 


\section{Supplementary}

\section{Flow cytometry staining and analysis}

(I) Marked flow pipe, $100 \mu \mathrm{L}$ blood samples $(50 \mu \mathrm{L}$ in absolute count tube) were added into each tube, and then fluorescein labeled cell membrane molecular antibodies were added according to the antibody instructions (Table S1), and incubated at $4{ }^{\circ} \mathrm{C}$ in dark for $20-30$ minutes.

(II) After the staining, $1 \mathrm{~mL}$ of red blood cell lysate was added into each tube (450ul in the absolute counter tube). After the oscillation, it was left for 10 minutes at room temperature and in dark, and then centrifuged at $300 \mathrm{~g}$ for 5 minutes (the absolute counter tube was directly put on the machine without centrifugation).

(III) The cells were resuspended with PBS and centrifuged at 300g for $5 \mathrm{~min}$. The cells were washed twice and added with 200 L PBS and prepared for loading.

i. Add an appropriate Staining Buffer. Adjust cell concentration to 106/100 L/Test;

ii. Labeled flow tubes were 1, 2, 3, 4, and 5 (Table S2). Each tube was labeled as shown in the table below. Fluorescein labeled cell membrane molecular antibodies were added according to the antibody instructions, and incubated at $4{ }^{\circ} \mathrm{C}$ in dark for $20-30$ minutes.

iii. After the staining, the cells were resuspended with PBS (300 percp-CY5.5) for 5 minutes, and then washed twice. Cells were resuspended with $200 \mathrm{~L}$ PBS (10 L 7AAD (percP-CY5.5 channel detection) active dye was added into each tube.

Table S1 Flow cytometry antibody labeling details

\begin{tabular}{|c|c|c|}
\hline Anti-bodies & Companies & Product No. \\
\hline CD4-PE & Biolegend & 317410 \\
\hline CD25-PE & Biolegend & 302606 \\
\hline CD127-APC & Biolegend & 351316 \\
\hline \multicolumn{3}{|l|}{ CD3-4-8, PD-1 } \\
\hline CD3-PE-cy7 & Biolegend & 344816 \\
\hline CD4-PE & Biolegend & 317410 \\
\hline CD8-FITC & Biolegend & 301050 \\
\hline PD-1-APC & Biolegend & 329908 \\
\hline CD3-FITC & Biolegend & 300306 \\
\hline CD56-PE & Biolegend & 362508 \\
\hline CD16-PE & Biolegend & 360704 \\
\hline CD19-APC & Biolegend & 302212 \\
\hline CD45-PERCP-CY5.5 & $\mathrm{BD}$ & 340953 \\
\hline Absolute counter tube & $\mathrm{BD}$ & 340334 \\
\hline
\end{tabular}


Table S2 Flow cytometry antibody labeling details

\begin{tabular}{lccccc}
\hline & FITC & PE & APC & PE-cy7 & APC-cy7 \\
\hline $1^{*}$ & CD4 & & IgG1-APC & CD3 & CD8 \\
2 & CD4 & & PD-L1 & CD3 & CD8 \\
$3^{*}$ & CD103 & IgG1 & PD-1 & CD3 & CD8 \\
$4^{*}$ & CD103 & TIM-3 & lgG1 & CD3 & CD3 \\
5 & CD103 & TIM-3 & PD-1 & CD8 \\
\hline
\end{tabular}

$1^{*}$, PD-L1 for continuous expression, the tube was set to a fluorescence minus one control (FMO) tube with no clustering; $3^{*}, 4^{*}$, Tim- 3 and PD-1, the cluster was not obvious in individual tumor tissues, and the two tubes were set as FMO tubes with no. 5 tubes.

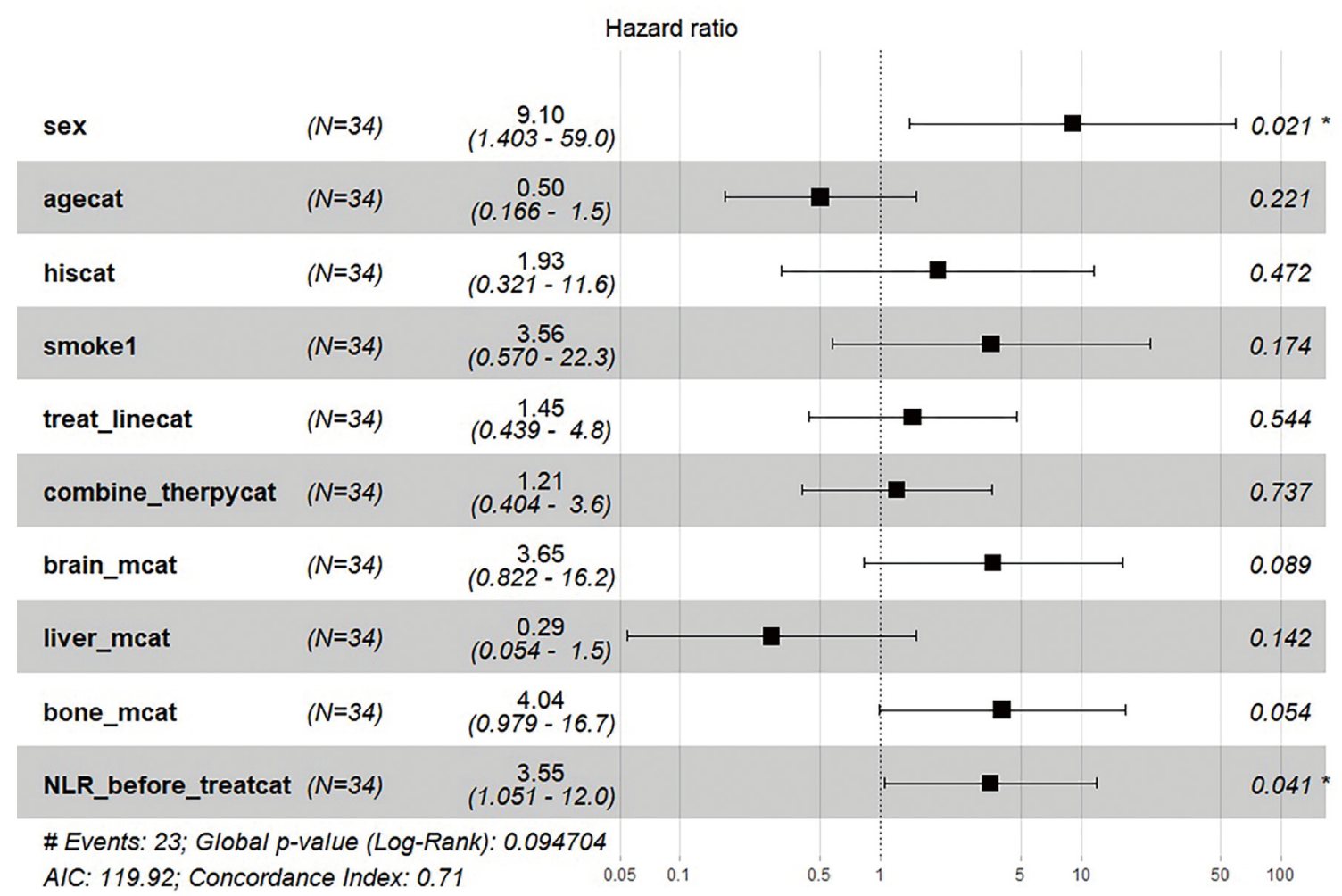

Figure S1 Forest plot for the associations between the 34 participant characteristics and PFS by Cox proportional hazards regression models. sex = gender; agecat = age category; smoke1 = smoker; treat_linecat $=$ treat line; combine_therapycat = treatment model; brain_mcat = brain metastasis; liver_mcat = liver metastasis; bone_mcat = bone metastasis; NLR_before_treatcat = NLR. 